Four of the 11 patients became seizure free for 5 to 20 years, 1 child remained seizure free for 12 months after viral infection, and 6 relapsed and had seizure recurrence within 14 days to 1 month after remission. Characteristic findings in the 4 patients with prolonged remission included: 1) normal brain CT/MRI, 2) normal development prior to onset of epilepsy, and 3) a short duration of epilepsy before occurrence of viral infection. One mechanism for seizure remission proposed is a change in immune state following viral infection similar to the effect of immunoglobulin therapy. Alternative hypotheses include: 1) direct suppression of seizures by virus, 2) elevation of serum cortisol level, or 3) anti-inflammatory cytokine response to viral infection. (Fujita Y, Imai Y, Ishii W et al. Improvement of intractable childhood epilepsy following acute viral infection. Brain Dev January 2011;33:62-68). (Respond: Dr Yukihiko Fujita, Nihon University School of Medicine, Tokyo, Japan. E-mail: yfujita@med.nihon-u.ac.jp).

COMMENT. Several reports of seizure remission following viral infection have involved patients with West syndrome, and some cases have a spontaneous remission. Among cases of viral-induced remission of epilepsy, exanthem subitum and human herpes virus-6 appear to be involved most commonly, and West syndrome is the most susceptible epilepsy. Previous reports of viral-induced seizure remission have described the clinical findings but have lacked the details of electrographic and hypsarrhythmia modification. The mechanism and therapeutic possibilities of this report deserve further study.

\title{
PROLONGED EEG DEPRESSION AS A PREDICTOR OF WEST SYNDROME IN TERM INFANTS WITH HIE
}

Seventeen term and near-term infants with hypoxic ischemic encephalopathy (HIE) were studied at Okazaki City Hospital and other centers in Japan to clarify the relationship between prolonged depression of the EEG and later development of West syndrome (WS). Group A, 4 patients with prolonged EEG depression over 21 days, and Group B, 13 with disappearance of EEG depression by 21 days of age, are compared. WS developed in all 4 infants in Group A, but only 1 of 13 in Group B. Abnormal irregular faster waves (irregular spiky beta or sharp theta) in 11 infants from both groups between 2 and 28 days of age were related to an adverse developmental outcome but not to West syndrome. Prolonged depression of EEG over 21 days of age in term infants with HIE is a predictor of later development of WS (sensitivity 0.80 , specificity 1.0 , pos predictive value of 1.0, neg predictive value 0.92). In comparison, MRI lesions in basal ganglia, thalamus, and white matter had a sensitivity of 0.80 , specificity 0.92 , pos predictive value of 0.80 , and negative predictive value of 0.92 . (Kato T, Okumura A, Hayakawa F et al. Prolonged EEG depression in term and near-term infants with hypoxic ischemic encephalopathy and later development of West syndrome. Epilepsia Dec 2010;51(12):2392-2396). (Respond: Toru Kato MD, Department of Pediatrics, Okazaki City Hospital, Okazaki, Aichi, 444-8553, Japan. E-mail: kato-jes@umin.ac.jp).

COMMENT. Watanabe and colleagues $(1987,2001)$ previously reported neonatal EEG abnormalities that preceded WS, but the predictive value of EEG depression was not established. The abnormal irregular faster waves, not significantly related to the later 
development of WS, were not precursors of hypsarrhythmia. Given the importance of early treatment of infantile spasms with ACTH for optimal benefit, the definition of a reliable pre-hypsarrhythmia pattern in the EEG of infants at risk of developing West syndrome should lead to more effective therapy and improved prognosis.

STXBP1 mutations account for one-third of cases of early infantile encephalopathy with suppression-burst pattern (Ohtahara syndrome) (Saitsu H et al. Epilepsia Dec 2010;51(12):2397-2405). Also, STXBP1 mutation should be considered as a possible cause of symptomatic West syndrome without known cause. (Otsuka M et al. Epilepsia Dec 2010;51(12):2449-2452).

\section{NEUROMUSCULAR DISORDERS}

\section{BENIGN COX DEFICIENCY MYOPATHY}

Clinical and pathological features of benign infantile mitochondrial cytochrome c oxidase $(\mathrm{COX})$ deficiency were studied in 8 patients with the disease phenotype seen at the National Center of Neurology and Psychiatry, Tokyo, Japan. Clinically, patients had severe generalized muscle hypotonia and weakness from birth to 3 months of age. Two patients had facial muscle involvement and high-arched palate. Prior to muscle biopsy and mitochondrial DNA analyses, including northern blot and respiratory chain enzyme activity assay, initial diagnoses were mitochondrial myopathy in 5 patients, congenital myopathy in 2, and congenital muscular dystrophy in 1 . All patients except 1 recovered completely during a 3-year follow-up period. All learned to walk by 1-2 years of age and could run at follow-up. Blood lactate levels were normal in 2 , slightly elevated in 4 , and >twice normal level in 2. Serum CK was elevated in all infants (182-644 U/L) and was normal at follow-up. Brain MRI was normal at age 7 months and 13 months but showed abnormally high T2-weighted signals in bilateral caudate nuclei and putamina at ages 23 and 33 months in 2 siblings; MRI was normal in 2 other patients examined. Muscle biopsy showed variation in fiber size, and numerous ragged-red fibers that were COX negative. Northern blot analysis revealed decreased levels of mitochondrial transfer RNA-glutamate molecules. All patients had a novel homoplastic m.14674T $>\mathrm{C}$ or $\mathrm{m} .14674 \mathrm{~T}>\mathrm{G}$ mutation as the site of disease causation, resulting in defects of COX and multiple respiratory chain enzymes. In addition to muscle, the basal ganglia may aiso be involved. Normal respiratory chain enzyme activities found in naïve myoblasts was evidence of potential for spontaneous recovery. (Mimaki M, Hatakeyama H, Komaki H, et al. Reversible infantile respiratory chain deficiency: a clinical and molecular study. Ann Neurol Dec 2010;68(6):845-854). (Respond: Dr Y Goto, National Institute of Neuroscience, Kodaira, Tokyo 187-8502, Japan. E-mail: goto@ncnp.go.jp).

COMMENT. The authors conclude that the pathogenicity of the mutation at np 14674 in tRNA, resulting in deficiency of multiple respiratory enzymes, is confirmed. Compensatory mechanisms result in variation of clinical features from asymptomatic to both muscle and CNS involvement. They propose a change in name to "infantile reversible respiratory chain deficiency" since multiple respiratory chain enzyme deficiency may result in more than myopathy alone. 